\title{
Dependence of neighbor data sequences based on Current Mode Controlled Boost Converter
}

\author{
Fangya Sun ${ }^{\mathrm{a}}$, Xitong Zhu ${ }^{\mathrm{b}}$, Haoshen $\mathrm{Li}^{\mathrm{c}}$, Yan Luo ${ }^{\mathrm{d}}$ and Hongmei $\mathrm{Xu}^{\mathrm{e}^{*}}$ \\ Institute of Engineering Yanbian University, Yanji, China

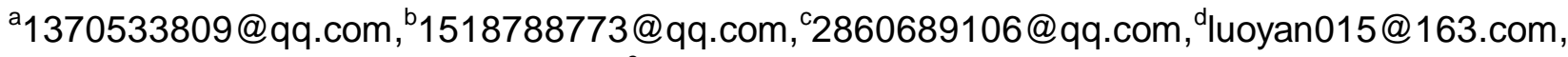 \\ ehmxu@ybu.edu.cn \\ * The corresponding author
}

Keywords: Boost converter; Chaos; Neighbor data; Relevance; Quantitative

\begin{abstract}
Based on the data relevance analysis method, the distance value of the adjacent data subsequences in the one-dimensional sequence of the current mode Boost converter is measured, converts complex numeric columns to a simpler range value sequence, by means of qualitative observation and quantitative analysis, the working status of current mode Boost converter is identified and quantified. The design of an actual applied circuit has certain instructions.
\end{abstract}

\section{Introduction}

The chaos inherent in nature and social life is constantly being revealed. The study of chaos is widespread in many fields, such as economy[1], medicine[2], transportation[3], information networks[4] and marine science[5]. The current mode controlled Boost converter has many advantages such as large gain, fast dynamic reaction, relatively small output inductance and relatively simplified compensation circuit[6]. The operation mode of the Boost converter is continuous current conduction mode and current interrupted conduction mode. Due to the uncertainty of chaotic motion, the operating state of the system cannot be predicted and controlled. Therefore, it is necessary to solve the problem in the field of power supply design to study the operation interval of the Boost switch converter when the parameter changes.

Ott E, Grebogy $\mathrm{C}$ and Yorke $\mathrm{J}$ A solved the problem of controlling chaotic behavior by means of small segmental time-varying parameters or dynamic incremental input[7]. In the literature [8], the calculation method of the lyapunov exponent of the chaotic Boost converter was systematically analyzed, and the characteristics of the chaotic state were analyzed, and the difference in the graph of the lyapunov exponent was compared. The analytical method of the nonlinear behavior of DC-DC converters using symbolic time sequence and entropy theory are proposed in literature[9], and calculated the module entropy of the symbol sequence using the informatics method, which leads to a quantifiable nonlinear dynamical index. The method of analyzing nonlinear behavior of DC-DC converters using symbolic time series and entropy theory are proposed in literature[10], which based on convert topological conjugation theory to the symbol sequence, the relative entropy of the symbol sequence is calculated by the forward sequence and backward sequence probability.

In this paper, a chaotic analysis method based on the correlation of adjacent data sequences is proposed, measuring changes in distance values of one-dimensional data subsequences of switching converters, convert complex data columns into simpler sequence of distance values, the process of phase space reconstruction and scale-free zone selection is avoided, and the qualitative and quantitative analysis methods are organically combined, it can clearly distinguish the different operating states of the switching converter, it provides an effective analysis method for the research of switching converters. 


\section{The Analysis Method of Data Relevance}

The characteristics of Euclidean distance are calculated by calculating the Euclidean distance of the contiguous subsequence in the one-dimensional data sequence. For data sequence, using Eq. 1 to calculate distance:

$$
d_{i}=\left|X_{i+1}-X_{i}\right|=\left(\sum_{j=0}^{m-1}\left(x_{i+1+m}-x_{i+m}\right)\right)^{1 / 2}
$$

In the equation, $i \leq n-m, d_{i}$ is the distance of adjacent subsequence. According to the change of value $i$, calculate the distance of adjacent subsequence in turn, and compose a new sequence $\left\{x_{d}\right\}$. Calculating the mean and standard deviation of new sequence to analyze nonlinear dynamic characteristic.

In order to analyze the data dependence, use the change rate and change range of adjacent points for further analysis, using Eq.2 and Eq.3:

$$
\begin{aligned}
& R_{j}=\left|d_{j+1}-d_{j}\right| / d_{j} \\
& V_{j}=\left|d_{j+1}-d_{j}\right|
\end{aligned}
$$

In the equations, $j \in[1, L], L$ is the length of adjacent subsequence, the change rate and change range form new sequence. The trend of the two changes can further analyze the data dynamic characteristics.

\section{Discrete Modeling and Relevance Analysis of Current Controlled Boost Converter}

In this paper, data correlation analysis is carried out based on the model of switch converter proposed by TSE, and the schematic diagram of current mode control Boost converter is shown in figure 1 .

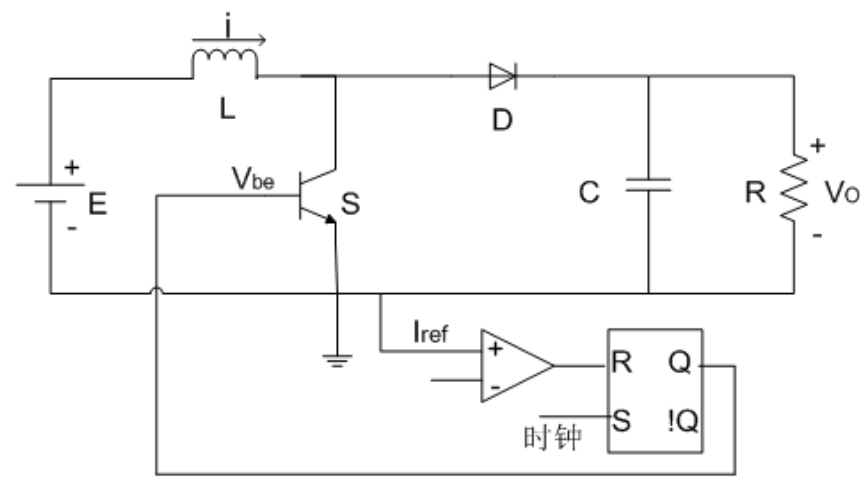

Figure 1. Current feedback Boost converter schematic diagram

$E$ is input voltage, $i$ is expected steady-state inductor curren, $R$ is load resistance, the working state of the converter can be described by the following iterative mapping equation.

$$
i_{n}=e^{-t_{m} / 2 R C}\left[\frac{c_{1}}{R}+c *\left(c_{2} w-\frac{C a_{1} c_{1}}{2}\right)\right] \cos w t_{m}+e^{-t_{m} / 2 R C}\left[\frac{c_{2}}{R}-C *\left(\frac{c_{2} a_{1}}{2}+c_{1} w\right) \sin w t_{m}\right]+\frac{E}{R}
$$

Other parameters are expressed as follows:

$$
w=\sqrt{\frac{1}{4 R^{2} C^{2}}-\frac{1}{L C}}
$$




$$
\begin{gathered}
c_{1}=v_{n} * e^{-t_{n} / R C}-E \\
c_{2}=\frac{\left(I_{r e f} R-\frac{c_{1}}{2-V I}\right)}{w R C}
\end{gathered}
$$

The parameters of the converter are shown in Table 1, and the bifurcation diagram of Boost converter is obtained: when $R<22$, the current mode controlled Boost converter works in periodic state; when $22 \Omega<R<34 \Omega$, the current mode controlled Boost converter works in two period; when $R>38 \Omega$, the current mode controlled Boost converter works in chaotic state as shown in figure 2, the phase diagrams of the two cycles and the chaotic phase are shown in fig. 3(a) and fig. 3(b).

\begin{tabular}{|c|c|c|c|}
\hline Parameter & Values & Parameter & Values \\
\hline Input voltage $\mathrm{E}[\mathrm{V}]$ & 10 & Capacitance $C[\mu F]$ & 12 \\
\hline Period & 100 & Inductance & 1 \\
\hline Reference current Iref[A] & 0.5 & & \\
\hline
\end{tabular}

Table 1 Boost Converter parameter

Load resistance $R$ is variable and take a hundred values of $R$ from $20 \Omega$ to $45 \Omega$, obtained a iteration sequence that data length is 5000 according to Eq.4, remove the first 1000 transient data of the original sequence, then calculating the adjacent subsequence distance composed adjacent subsequence distance sequence according to the Eq.1, calculating the mean value and standard deviation of the adjacent distance sequence respectively, as shown in Fig. 4 and Fig. 5.

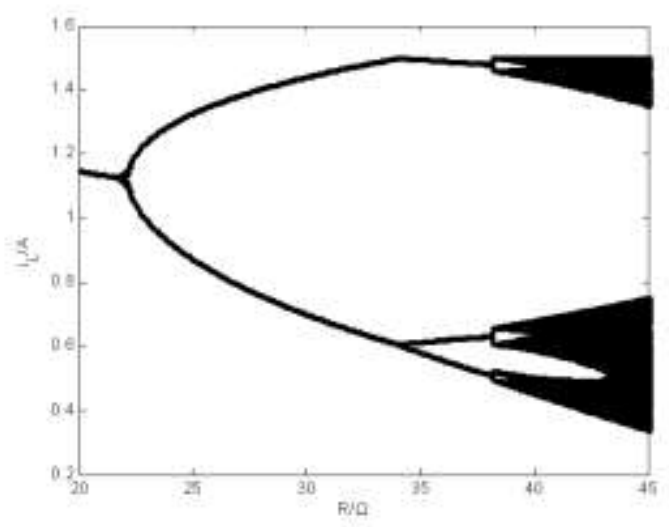

Figure 2. Current feedback Boost converter bifurcation diagram

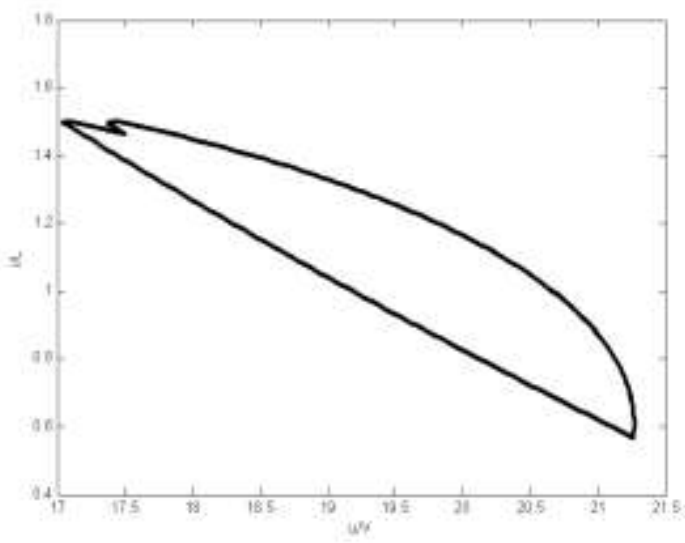

Figure 3(a). Two-phase phase diagram

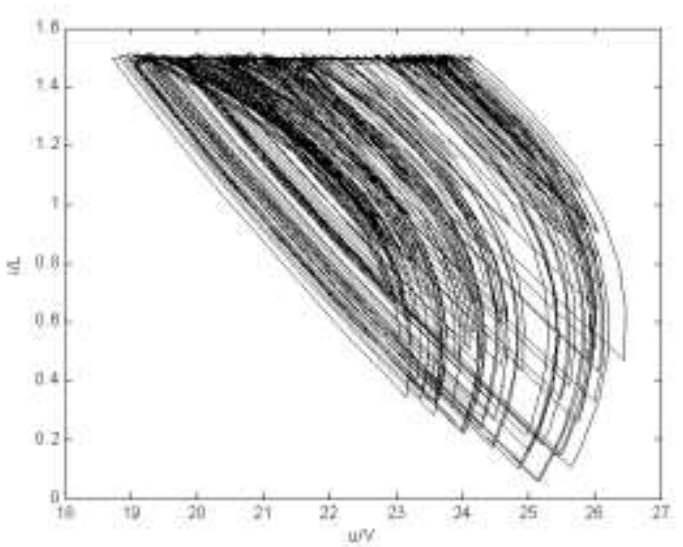

Figure 3(b). Chaotic Phase diagram 
When the switching converter appears bifurcation phenomenon, the adjacent distance value increases with the smooth curve, at the bifurcation, the average value oscillates accordingly, and after entering the chaos, a smaller peak value appears as shown in Fig. 4. The standard deviation value is significantly greater than 0 when the switching converter enters chaotic state, and it shows an increasing trend in significant fluctuations as shown in Fig. 5. Corresponding to the bifurcation diagram, the standard deviation value can clearly quantify the change of the chaotic characteristics.
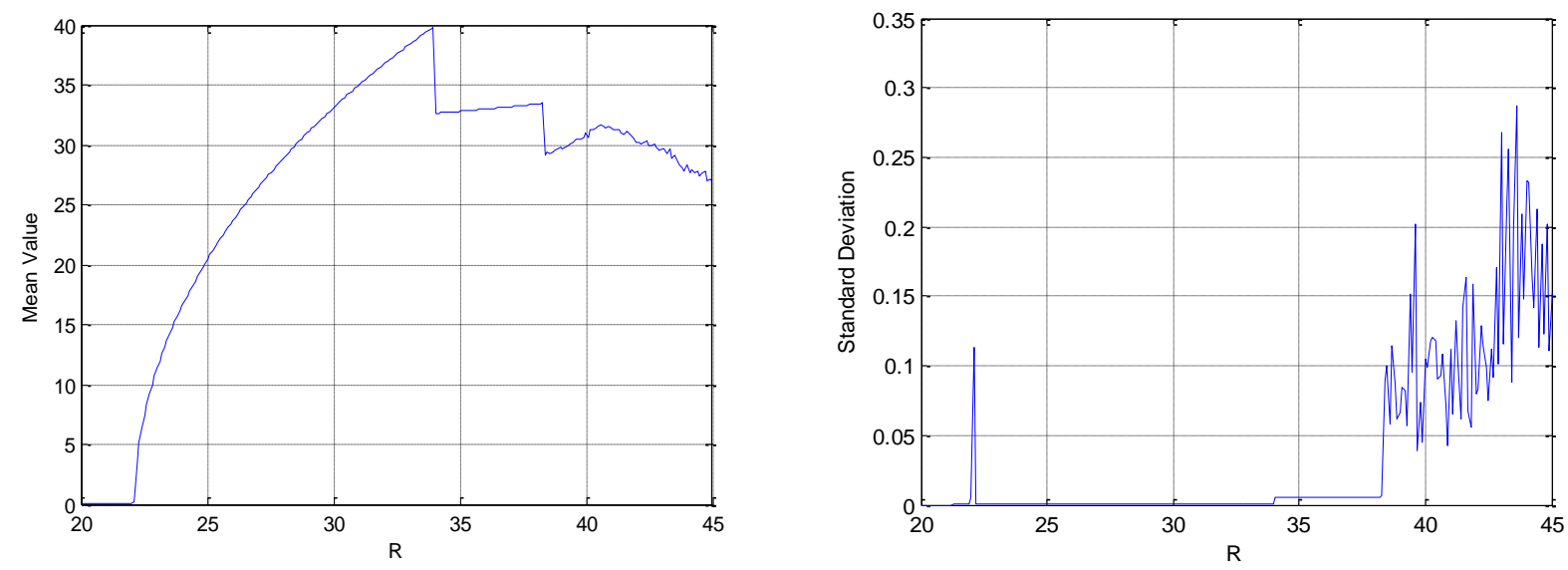

Figure 4. Adjacent distance sequence mean value curve Figure 5. Adjacent distance sequence standard deviation value curve

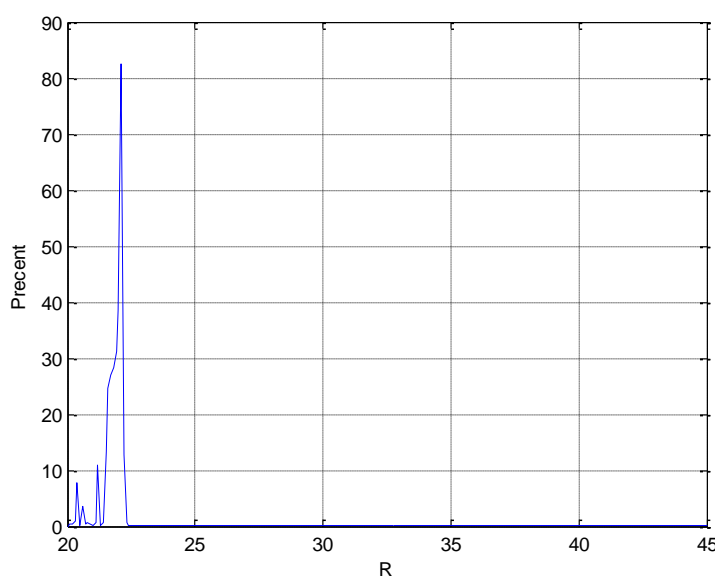

Figure 6. Adjacent point change rate curve of adjacent distance mean sequence

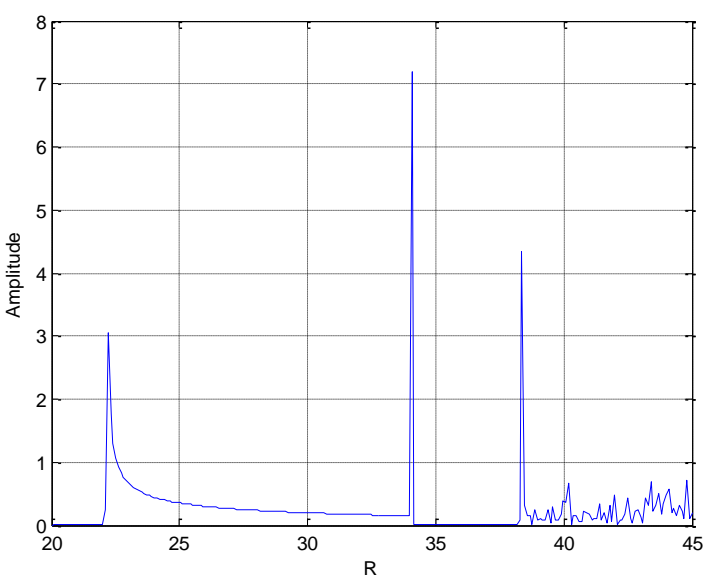

Figure 7. Adjacent point change range curve of adjacent distance mean sequence

The change rate and range of adjacent points can be obtained according to Eq.(2) and Eq.(3). When the operating state of the switching converter enters two cycles, there is a clear peak in the rate of change and the magnitude of changes in adjacent points. When entering the chaotic state, a series smaller peaks and valleys appears in the amplitude of the adjacent points as shown in Fig. 6 and Fig. 7. Therefore, the analysis method based on the correlation of adjacent data has the ability to quantify and is sensitive to changes in chaotic characteristics.

\section{Conclusions}

Based on the current mode controlled Boost Converter as an example, the following conclusions are obtained by analyzing the distance value variation between adjacent data sequences: With the change of selected parameter, the mean of adjacent distance can clearly describe the process from bifurcation and period-doubling to chaotic state. The standard deviation of adjacent distances can identify and quantify its corresponding operating range and determine the corresponding chaotic state. The change rate and 
variation range of data points in adjacent distance sequences can further improve the quantitative analysis of switching converters. Compared with existing analytical methods, the method has a one-to-one correspondence between the parameter variation range and the current mode controlled Boost converter operating status. The results of this paper quantified the chaotic characteristics of the circuit. For the actual circuit analysis and design provide an effective theoretical basis.

\section{References}

[1] Zhang X D, Liu X D, Zheng Y, Liu C: Chin. Phys. B, 2013, No.22, p.030509.

[2] Coelho A L V, Lima C A M, Eng. Appl. Artif. Intel, 2014, No.36, p.81.

[3] Krese B, Govekar E, Transport. Res. C, 2013, No.36, p.27.

[4] Wang J S, Yuan R X, Gao Z W, Wang D J, Chin. Phys. B, 2011, No.20, p.090506.

[5] Mohammad Z K, Ozgur K, Ocean Eng. 2015, No.100, p.46.

[6] Axelrod B, Berkovich Y and Ioinovici A, IEEE Trans. Circuits and Systems. I, Vol. 55 (2008) No.2, p.687.

[7] Bao B C, Xu J P and Liu Z, Chinese Physics B, Vol. 18 (2009), No.11, p.4742.

[8] Zhou G H, Xu J P, Bao B C, Zhang F, Liu X S, CHIN. PHYS. LETT, Vol. 27 (2010) No.9, p.090504-1.

[9] Letellier C, Chaos, Solitons\&Fractals. 2008, No.36, p.32.

[10] Venkat Chandrasekaran, Parikshit Shah, Mathematical Programming, Vol.1-2 (2017) p.161. 